\title{
There is no ambiguity in the radiatively induced gravitational Chern-Simons term
}

\author{
Brett Altschul* \\ Department of Physics and Astronomy, University of South Carolina, \\ Columbia, South Carolina 29208, USA
}

(Received 24 March 2019; published 18 June 2019)

\begin{abstract}
Quantum corrections to Lorentz- and $C P T$-violating QED in flat spacetime produce unusual radiative corrections, which can be finite but of undetermined magnitude. The corresponding radiative corrections in a gravitational theory are even stranger, since the term in the fermion action involving a preferred axial vector $b^{\mu}$ would give rise to a gravitational Chern-Simons term that is proportional $b^{\mu}$, yet which actually does not break Lorentz invariance. Initially, the coefficient of this gravitational Chern-Simons term appears to have the same ambiguity as the coefficient for the analogous term in QED. However, this puzzle is resolved by the fact that the gravitational theory has more stringent gauge invariance requirements. Lorentz symmetry in a metric theory of gravity can only be broken spontaneously, and when the vector $b^{\mu}$ arises from spontaneous symmetry breaking, these specific radiative corrections are no longer ambiguous but instead must vanish identically.
\end{abstract}

DOI: $10.1103 /$ PhysRevD.99.125009

\section{INTRODUCTION}

Since the 1990s, there had been a significant renewal of interest in the possibility that the seemingly absolute Lorentz and $C P T$ symmetries of the standard model and gravity might actually be very weakly violated. At this time, there is no compelling evidence for such symmetry breaking. However, if violations of isotropy, Lorentz boost invariance, or $C P T$ were ever observed experimentally, the discovery would obviously be of fundamental importance. It would change our understanding of how physics works at the very deepest levels. Even if there is no current evidence for Lorentz or $C P T$ violation, these symmetries are so basic (as fundamental building blocks of both quantum field theories and the general theory of relativity) that they are worthy of careful study.

There are also other reasons to be interested in Lorentz and $C P T$ tests. Attempts to develop a quantum theory of gravity have shown that many of the speculative frameworks that have been suggested to describe quantum gravity seem to allow for the existence of Lorentz violation, at least within certain regimes. Moreover, with the explication of a comprehensive effective field theory (EFT) capable of describing Lorentz-violating phenomena, it

\footnotetext{
*baltschu@physics.sc.edu
}

Published by the American Physical Society under the terms of the Creative Commons Attribution 4.0 International license. Further distribution of this work must maintain attribution to the author(s) and the published article's title, journal citation, and DOI. Funded by SCOAP. came to be realized that the symmetry violations could come in a much wider variety of forms than previous unsystematic analyses had considered. Large regions of the EFT parameter space were scarcely constrained by earlier generations of experiments. $C P T$ symmetry is also closely tied to Lorentz symmetry, so that even with nonlocal interactions, $C P T$ violation in a quantum field theory (QFT) automatically entails Lorentz violation [1], as long as the theory has a well-defined $S$-matrix.

The general EFT describing Lorentz violation in particle physics therefore includes the most general $C P T$ violation as well. This EFT is known as the standard model extension (SME) [2,3]. The action for the SME contains operators that can be constructed out of the standard model fields. The usual standard model action is formed by writing down all the local, renormalizable, $S U(3)_{c} \times S U(2)_{L} \times U(1)_{Y}$ gauge-invariant, Lorentz-invariant operators that can be constructed from those fields. The SME is constructed in much the same way, but with the Lorentz invariance requirement dropped; specifically, the minimal SME (mSME) keeps all of the other requirements-locality, renormalizability, and gauge invariance. The mSME is now the usual framework used for parametrizing the results of experimental Lorentz and $C P T$ tests. However, since the mSME action contains quite a large number of parameters, many different types of experiments have turned out to be useful for establishing bounds on the mSME parameters. An up-to-date summary of the results of these experiments is given in [4].

Studies of possible Lorentz and $C P T$ violation have also been fruitful theoretically, providing new insights, especially 
into the structure of QFTs. The radiative corrections to the Chern-Simons term in Lorentz-violating quantum electrodynamics (QED) have been one of the most studied topics related to the SME - and almost certainly the most controversial.

There is a similarly structured gravitational ChernSimons term in the gravitational version of the SME. Although the radiative corrections in the gravitational sector have been examined to a limited extent, a profound and significant puzzle exists in that sector-which has previously been overlooked. This paper will both introduce this puzzle and proceed to solve it.

\section{THE PUZZLE OF GRAVITATIONAL RADIATIVE CORRECTIONS}

The basic outline of the puzzle is the following. We must begin with a discussion of the simpler electromagnetic Lorentz violation term. The Chern-Simons term in the $(3+1)$-dimensional Abelian gauge sector takes the form $\mathcal{L}_{A F}=\frac{1}{2} k_{A F}^{\mu} \epsilon_{\mu \alpha \beta \gamma} F^{\alpha \beta} A^{\gamma}$ [5]. (When $k_{A F}^{\mu}$ is purely timelike, this term in the Lagrange density is proportional to $\vec{A} \cdot \vec{B}$, which breaks $P$ and $C P T$ symmetries.) Coming from the charged fermion sector, there is a radiative correction to $\mathcal{L}_{A F}$ that is necessarily finite, but whose coefficient is undetermined. At the quantum level, there is an infinite family of different theories that correspond to the same classical Lagrangian. The differences between these theories are in how they are regulated, but there is nothing that singles out one regulator as being unambiguously correct. Different high-momentum regulators lead to radiatively generated terms with different finite coefficients [6-21]. Various schemes have been suggested for identifying a single correct result; some authors have argued that only their certain specific regulators were appropriate for the calculation-and thus that there was one true correct answer. However, all such unambiguous answers appear to suffer from one of two deficiencies. The most naive schemes fix the term by demanding that the Lagrange density be gauge invariant; since the Chern-Simons term is not gauge invariant (it changes by a total derivative under a gauge transformation), the term is automatically ruled out. However, this is not a legitimate result, because it excludes the term of interest a priori. Gauge invariance of the Lagrange density is an unnecessarily strong condition; if we instead only demand that the integrated action be invariant, the Chern-Simons term is fully allowed. Alternatively, nonperturbative schemes for fixing the radiative correction have also been suggested. However, for a nonperturbative framework to make sense, it must provide a way of determining higher-order radiative corrections as well as first-order ones; and unfortunately, all the proposed nonperturbative methodologies that lead to particular nonzero values of the induced Chern-Simons coefficient appear to fail at higher order.
Calculations appear to show that the gravitational sector has the same kind of ambiguity [22-26]. The Lorentzviolating Chern-Simons term for $(3+1)$-dimension gravity is

$$
\mathcal{L}_{\Gamma}=-\frac{1}{4} v_{\mu} \epsilon^{\mu \alpha \beta \gamma}\left(\Gamma_{\alpha \tau}^{\sigma} \partial_{\beta} \Gamma_{\gamma \sigma}^{\tau}+\frac{2}{3} \Gamma_{\alpha \tau}^{\sigma} \Gamma_{\beta \eta}^{\tau} \Gamma_{\gamma \sigma}^{\eta}\right),
$$

in terms of the Christoffel symbols $\Gamma_{\alpha \beta}^{\gamma}$ [and with $\left.\kappa=(8 \pi G)^{-1}=1\right]$. In the linearized gravity limit, this may be expressed more conveniently directly in terms of the metric fluctuations. $\mathcal{L}_{\Gamma}$ is proportional to $v^{\mu} \epsilon_{\mu \alpha \beta \gamma} h^{\beta \nu} \partial^{\gamma}\left(\partial_{\sigma} \partial^{\sigma} h_{\nu}^{\alpha}-\partial_{\nu} \partial_{\sigma} h^{\alpha \sigma}\right)$, where $g^{\mu \nu}=\eta^{\mu \nu}+h^{\mu \nu}$. Not surprisingly, the gravitational Chern-Simons term contains two more derivatives than the electromagnetic term (to match the number of free $h^{\mu \nu}$ indices); but otherwise the two types of terms appear (in the weak field limit) to be quite similar in structure. However, there is actually a fundamental difference between the electromagnetic Chern-Simons term that may be radiatively generated in Lorentz-violating QED and the corresponding gravitational Chern-Simons term. The difference is that the gravitational Chern-Simons term is, in spite of appearances, actually Lorentz invariant. The profound puzzle that faces us is that it appears to be possible for a Lorentz-violating $b^{\mu}$ term in the fermion sector to generate a radiative correction that is proportional to $b^{\mu}$ and fully $P$ violating [27-29], yet which is invariant under all rotations and Lorentz boosts.

The Lorentz invariance of a pure gravity theory that includes a Chern-Simons term is rather subtle. In fact, this was itself a bit of a puzzle when the term was first introduced [30]; it appeared that there were no physical distinctions between versions of the theory with explicit (externally imposed) symmetry breaking and certain types of dynamical symmetry breaking. However, this was ultimately explained, and the Lorentz symmetry of the gravitational Chern-Simons theory was demonstrated by constructing the conserved gravitational energymomentum (pseudo)tensor $\Theta^{\mu \nu}$ [31]. This $\Theta^{\mu \nu}=\Theta^{\nu \mu}$ has a symmetric form, and symmetry of the energy-momentum tensor is equivalent to Lorentz invariance of the $S$-matrix (because the rotation and boost generators can be expressed as integrals of moments of $\Theta^{\mu \nu}$ ). Evidently, the dependence of the theory on the preferred vector $v^{\mu}$ is illusory. It is not possible to write down such a Chern-Simons term without introducing such a vector, but the particular spacetime direction of $v^{\mu}$ turns out to have no bearing on the physics. This is directly related to the gauge invariance of the theory; the semblance of Lorentz violation is essentially a gauge artifact.

Nonetheless, the gravitational Chern-Simons term really does break the discrete symmetries of general relativity. For a timelike $v^{\mu}$, the boost violation that is seemingly apparent in the form of the term is unphysical, but the parity violation is quite real. Boost invariance manifests itself in 
the fact that all gravitational waves in the theory propagate at the speed of light. However, the $P$ breaking means that right- and left-polarized waves are coupled to their sources with different strengths. Note that the lack of Lorentz violation in the $C P T$-violating gravitational Chern-Simons theory demonstrates that $C P T$ violation in the gravitational sector does not automatically need to be accompanied by Lorentz violation. This is a somewhat surprising result, although it is clear upon careful reinspection that the formal derivation [1] of the result that $C P T$ violation requires Lorentz violation does not technically apply in the context of a metric theory of gravity. Purely gravitational theories are not formulated using QFT to begin with, and stability of the quantum vacuum (required for the definition of the $S$-matrix) is thus not a condition that can formally be applied. Physically, this corresponds to the fact that there is generally no reason to expect that matter in a universe cannot progressively coalesce into heavier and heavier black holes, there being no ultimately stable lowest-energy configuration.

So while $b^{\mu}$ and $v^{\mu}$ terms have the same discrete symmetries, $b^{\mu}$ breaks Lorentz invariance, while $v^{\mu}$ does not. Returning to the main problematical observation, it appears that if a fermion species with a Lorentz-violating $b^{\mu}$ term is coupled to gravity, radiative corrections may produce a $\mathcal{L}_{\Gamma}$ term with coefficient $v^{\mu}$ proportional to $b^{\mu}$. The radiative correction would thus possess a much greater degree of symmetry than the novel term that generated it. It is not immediately clear whether this is possible or whether it should be ruled out by some general principles of field theory. Whichever option is correct, there is evidently quite a bit more to be understood about how radiative corrections work in these kinds of theories.

Having established the existence of this open question, we shall show that the resolution of this enigma is rather subtle. The key pieces of information necessary to construct the solution are embedded in the theory, but they need to be pieced together, in conjunction with what is already known about the general structure of Lorentz-violating field theories. The ultimate answer will be tied to the fact that gravitational theories are fundamentally different from other field theories when it comes to Lorentz violation. In particular, Lorentz violation in a metric theory must arise spontaneously.

\section{STRUCTURE OF AMBIGUOUS CORRECTIONS}

The fact that Lorentz symmetry in a metric theory of gravity can only be broken spontaneously will have profound consequences for the radiative corrections to the gravitational Chern-Simons term. To understand these consequences, we must look very carefully at the structures of both the electromagnetic and gravitational Chern-Simons terms. This will reveal a close connection to chiral anomalies.

\section{A. Abelian theory}

The QED Lagrange density, including the only mSME term that has the right structure to make a radiative contribution to $\mathcal{L}_{A F}$, is

$$
\mathcal{L}_{\mathrm{QED}}=-\frac{1}{4} F^{\mu \nu} F_{\mu \nu}+\bar{\psi}\left(i \not-m-q \not A+\not \phi \gamma_{5}\right) \psi
$$

The pair of diagrams that contribute to the undetermined radiative correction in an Abelian gauge theory is shown in Fig. 1(a). These are essentially just the same diagramswith fermion triangles and two external photons- that are responsible for the chiral anomaly.

The two diagrams in Fig. 1(a) differ in the direction of the fermion number flow around the triangular loop. Alternatively (taking the viewpoint suggested by the nonperturbative treatment in [7]), there is just a single one-loop diagram - the usual vacuum polarization diagram, but with the modified fermion propagator

$S_{b}(l)=\frac{i}{l-m+\not b \gamma_{5}} \approx \frac{i}{l-m}+\frac{i}{l-m}\left(i \not b \gamma_{5}\right) \frac{i}{l-m}$.

(Since the Lagrange density involves no nonstandard time derivatives, the fermion sector may be quantized without any changes to the spinor representation [32,33], and the $b^{\mu}$-exact propagator may simply be read off from $\mathcal{L}_{\mathrm{QED}}$.) The two triangles then arise from the fact that, at first order
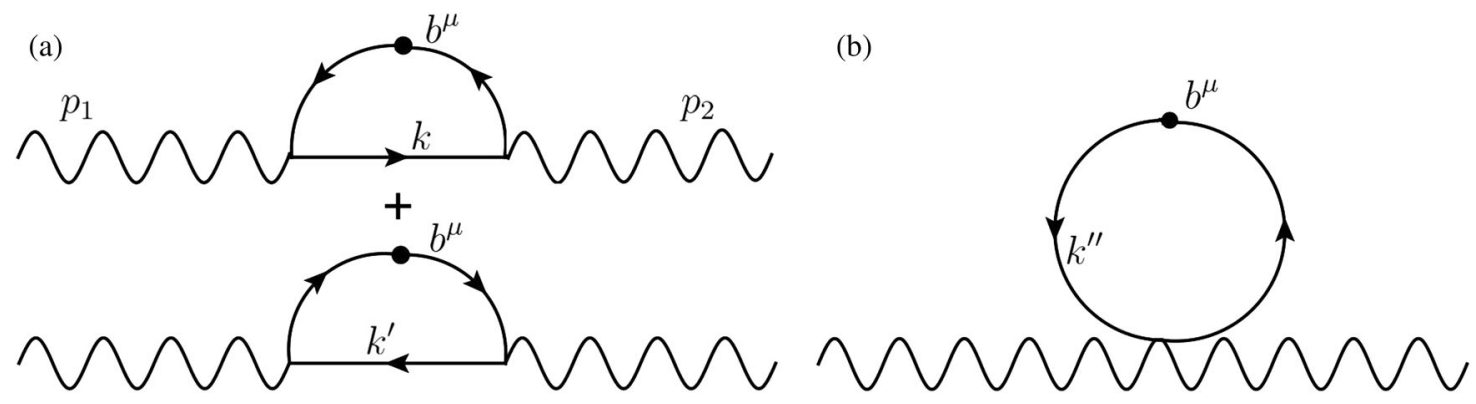

FIG. 1. One-loop diagrams that can contribute to the radiatively generated Lorentz-violating Chern-Simons terms. The dots represent the $b^{\mu}$ insertions appearing in the fermion propagator $S_{b}$. (a) The two triangle diagrams that exist in the radiative calculation of the Abelian Chern-Simons term. (b) The additional contributing diagram that appears in the gravitational theory. 
in the Lorentz violation, there may be a $b^{\mu}$ insertion on exactly one of the two internal fermion lines.

The two triangle diagrams are very similar to those that arise in the calculation of the QED chiral anomaly-for example, in its original context of $\pi^{0}$ decay [34]. Each triangle has two vertices attached to outgoing gauge boson propagators, and a third axial vector vertex with a $\gamma_{5}$. The presence of the $\gamma_{5}$ is what ensures that, when the loop momentum is very large, the contributions from the two different diagrams cancel out, since the fields passing through the $\gamma_{5}$ vertex have opposite chiralities. A great deal is known about the structure of these kinds of diagrams, from analyses of the chiral anomaly.

However, there is a subtle but fundamental difference between how the sum of the two triangle diagrams should be evaluated, in the contexts of Lorentz violation versus $\pi^{0}$ decay. The issue is that, when a meson vertex is involved, there is an additional leg attached there, which represents the incoming decaying particle. That particle carries momentum, so the two fermion propagators attached to that vertex will have different momenta. In contrast, in a theory with explicit Lorentz violation, the $b^{\mu}$ vertex cannot carry any momentum whatsoever, because $b^{\mu}$ is constant across all spacetime. Surprisingly, this changes the way the calculations can proceed in a significant way.

It was initially argued [6] — incorrectly — that the triangle diagrams could not generate a Chern-Simons term, because of the theory's gauge invariance properties. In each diagram, there are external photons attached to two of the triangle's corners, carrying momenta $p_{1}$ and $p_{2}$. Ward identities then imply that the amplitude $\mathcal{M}_{\mu \nu}$ corresponding to the sum of the two fermion loops must be transverse to both $p_{1}$ and to $p_{2}$. That is,

$$
\mathcal{M}_{\mu \nu} p_{1}^{\mu}=\mathcal{M}_{\mu \nu} p_{2}^{\nu}=0 .
$$

The implies that the amplitude must be $\mathcal{O}\left(p_{1}\right)$ and separately $\mathcal{O}\left(p_{2}\right)$. If $p_{1}$ and $p_{2}$ are allowed to be different (that is, if the axial vector vertex can carry a nonzero momentum), this implies that $\mathcal{M}^{\mu \nu}$ is $\mathcal{O}\left(p_{1} p_{2}\right)$. When we set $p_{1}=-p_{2}$, corresponding to the physical situation, the amplitude must be $\mathcal{O}\left(p_{1}^{2}\right)$. Since the Chern-Simons term is only $\mathcal{O}(p)$, it would appear that the Abelian Chern-Simons term cannot be generated by radiative corrections. However, this simple argument fails when there is no momentum input at the fermion triangles' third vertices. If $p_{1}$ is always identically equal to $-p_{2}$, then the two transversality conditions in (4) are redundant, and the matrix element only needs to be $\mathcal{O}\left(p_{1}\right)$, meaning a Chern-Simons term is actually allowed.

Without two independent Ward identities to be satisfied, the sum of the two triangle diagrams is actually undetermined, because the diagrams are each naively linear divergent, and there is no unique way to regulate them. However they are regulated, the divergent parts of the two diagrams will cancel, producing a finite result. A specific regulator is often most conveniently expressed in terms of a relationship between the loop momenta $k$ and $k^{\prime}$ in the two diagrams. If the amplitude really had needed to be transverse to two different photon momenta $p_{1}$ and $p_{2}$, it would have been necessary to choose $k^{\prime}=k+3 p_{1}$ and then (after Wick rotation) to perform a spherically symmetric integration over $k$. This is why when the axial vector vertex represents a physical $\pi^{0}$-which carries a nonvanishing momentum - the chiral anomaly gives a unique result for the meson decay rate. However, when only a single transversality condition is imposed, it is possible to have $k^{\prime}=k+(3+\xi) p_{1}$ for any real value of $\xi$. While the induced Chern-Simons term vanishes for $\xi=0$, with nonzero values of $\xi$ there is a $k_{A F}^{\mu}=-\xi q^{2} b^{\mu} / 16 \pi^{2}$ proportional to $\xi$ [7]. Each value of $\xi$ essentially defines a different quantum theory, all based on the same classical Lagrangian. Lorentz- and $C P T$-violating QED is thus an example of a QFT with finite but undetermined radiative corrections; some general characteristics of such theories are discussed in [35].

With a momentum cutoff regulator, the shift in the integration by $\xi p_{1}$ produces a surface term, which is allowed to be nonzero because the full diagram is divergent. This kind of surface term is well known to create problems with gauge invariance. However, because of the presence of the $\gamma_{5}$ in the fermion loop, there must be a Levi-Civita tensor $\epsilon_{\mu \alpha \beta \gamma}$ in the resulting radiative correction to the photon two-point function; and because of the total antisymmetry of $\epsilon_{\mu \alpha \beta \gamma}$, the radiative correction (i.e., the induced Chern-Simons term $\mathcal{L}_{A F}$ ) still obeys the Ward identity. This is what ensures that the integrated action remains gauge invariant, even though gauge invariance is lost at the level of the Lagrange density.

Since surface terms are involved, it seems like it might be possible to avoid the Chern-Simons ambiguity by using a better regulator. However, both Pauli-Villars and dimensional regularization-normally the best choices when there are potential problems with maintaining gauge invariance-reintroduce the ambiguity in other ways. The Pauli-Villars method entails introducing additional families of fictitious heavy fermions, whose contributions to the photon self-energy are subtractive. However, the new fermions will posses their own $b^{\mu}$ terms, whose sizes are not determined by the classical Lagrangian. In dimensional regularization, there is no unique extension of $\gamma_{5}$ to $4-\epsilon$ dimensions, and different extensions will produce different Chern-Simons terms. With other regulation methods, the source of the radiative ambiguity may sometimes be disguised, but the ambiguity always appears to be present somewhere.

Several specific nonzero values for the induced $k_{A F}^{\mu}$ were suggested in the literature. These were typically based on various nonperturbative arguments for how the momentum integrations in the two triangle diagrams should be 
performed. However, any nonperturbative method should determine the structure of the radiative corrections not just at $\mathcal{O}(b)$, but also at $\mathcal{O}\left(b^{2}\right)$. It turns out that any choice of regulator that gives a specific nonzero coefficient for the Chern-Simons term at first order also breaks gauge invariance by producing a Lorentz-violating photon mass [36] term at second order in $b^{\mu}$ (e.g., $[20,21,25]$ ). The one natural exception is a regulator that produces a vanishing $k_{A F}^{\mu}$; it is always possible to enforce the maximal degree of gauge invariance at first order without spoiling gauge symmetry at higher orders.

\section{B. Gravitational theory}

Having pointed out all the key properties of the ambiguity in the Abelian Chern-Simons term in Lorentzviolating QED, we now turn our attention to the even trickier case of the gravitational Chern-Simons term. form

The gravitational SME action includes the $b^{\mu}$ term in the

$$
S_{\psi}=\int d^{4} x e e^{\mu}{ }_{a} \bar{\psi}\left(\frac{i}{2} \gamma^{a} \stackrel{\leftrightarrow}{D}_{\mu}+b_{\mu} \gamma^{a} \gamma_{5}\right) \psi
$$

where the fermions are taken to be massless (purely for simplicity). The vierbein (tetrad) is $e_{a}^{\mu}$, and its determinant is $e$. The coupling to gravitation occurs through $e^{\mu}{ }_{a}$ and through the gravitational covariant derivative, which is

$$
D_{\mu} \psi=\partial_{\mu} \psi+\frac{1}{2} \omega_{\mu c d} \sigma^{c d} \psi
$$

with the usual spin connection $\omega_{\mu}{ }^{c d}$ including derivatives of the vierbein. Because of the required vierbein factors, (5) is written as an integrated action $S$, although for linearized gravity it would actually be sufficient to work with just a Lagrange density. In the linearized theory and in harmonic gauge, chosen for its simplicity and convenience, especially when dealing with gravitational anomalies [37], the vierbein has a very simple representation in terms of the metric: $e_{\mu a}=\eta_{\mu a}+\frac{1}{2} h_{\mu a}$ and $e^{\mu}{ }_{a}=\eta^{\mu}{ }_{a}-\frac{1}{2} h^{\mu}{ }_{a}$.

Neglecting $h^{\mu}{ }_{\mu}$ interactions, which cannot contribute to the gravitational Chern-Simons term, the linearized Lagrange density for the fermions coupled to gravity is

$$
\begin{aligned}
\mathcal{L}_{\psi}= & \bar{\psi}\left\{\frac{i}{2}\left(\gamma^{\mu}-\frac{1}{2} h^{\mu \nu} \gamma_{\nu}\right) \stackrel{\leftrightarrow}{\partial}_{\mu}\right. \\
& \left.-h_{\mu \nu}\left[\frac{1}{2} b^{\mu} \gamma^{\nu} \gamma_{5}+\frac{i}{96}\left(\partial_{\rho} h_{\alpha \beta}\right) \eta^{\beta \nu} \gamma^{(\nu \beta \rho)}\right]+\not \not \gamma_{5}\right\} \psi .
\end{aligned}
$$

This expression involves the antisymmetrized product of three distinct $\gamma$-matrices, $\gamma^{(\nu \beta \rho)}=\gamma^{\nu} \gamma^{\beta} \gamma^{\rho} \pm$ (all permutations).

The corresponding Feynman rules for the perturbative interactions of gravitons with Lorentz-violating fermions
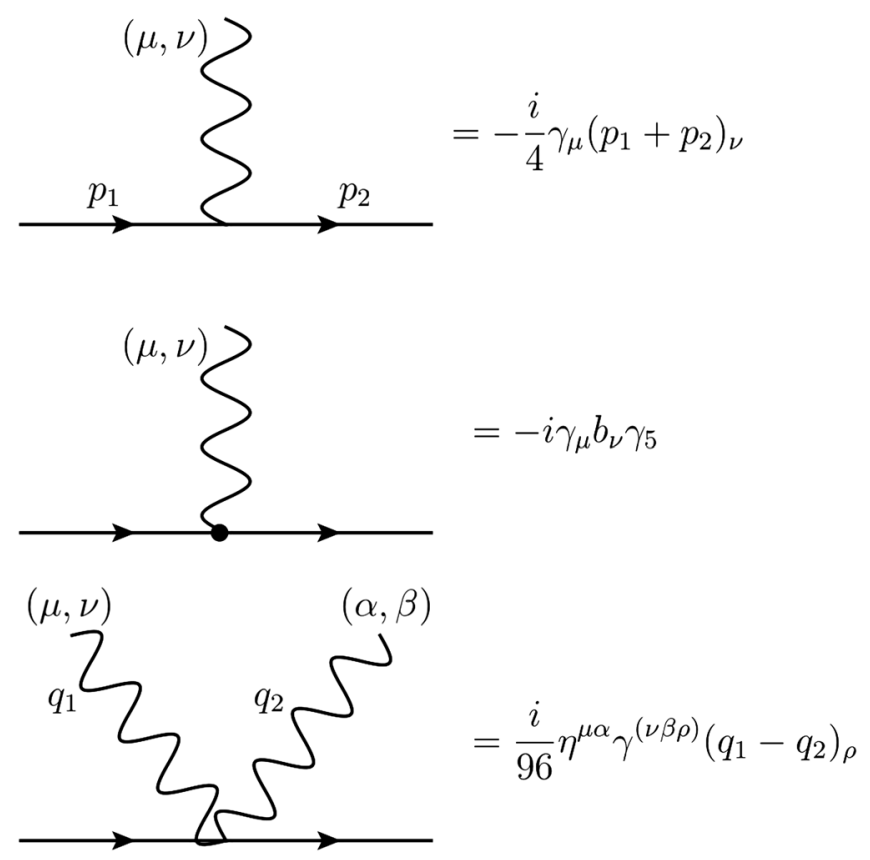

FIG. 2. Feynman rules for the fermion-graviton vertices in the presence of $b^{\mu}$.

are given in Fig. 2. There are the usual vertices for single or paired gravitational excitations $h^{\mu \nu}$ interacting with a fermion line, and there is also a new vertex in which $b^{\mu}$ appears. The new vertex exists because of the presence of $b^{\mu}$ in the energy-momentum tensor for the fermionic sector. However, it turns out that the new vertex does not actually make any contribution to the radiatively induced gravitational Chern-Simons term. On the other hand, both the usual three-particle vertex and the four-particle vertex involving $\gamma^{(\nu \beta \rho)}$ play potentially important roles.

All the two-point graviton diagrams derived from $\mathcal{L}_{\psi}$ that have a single fermion loop are shown in Figs. 1 and 3. However, only those in Fig. 1 can actually contribute to the Chern-Simons term (see below for details), and we shall therefore concentrate our attention on those three diagrams. Besides the presence of an additional diagram with a twograviton vertex, there is another way in which the gravitational radiative corrections are more complicated than those in the Abelian theory. Because the metric modes couple to the fermions' energy momentum, there are additional factors of the loop momentum appearing at the fermion-boson vertices. This gives the two triangle diagrams in Fig. 1(a) a naive cubic degree of divergence. The new diagram with the two-graviton vertex would also have a cubic divergence if the axial vector vertex could carry a nonzero momentum. However, with only a strictly constant $b^{\mu}$ inserted into the fermion propagator, the degree of divergence is reduced. In order to obtain a finite final results for the induced $v^{\mu}$, both the cubic and linear divergences in the sum of the diagrams must be canceled.

The more elaborate cancellation is generally possible (and necessary in order to preserve gravitational gauge 


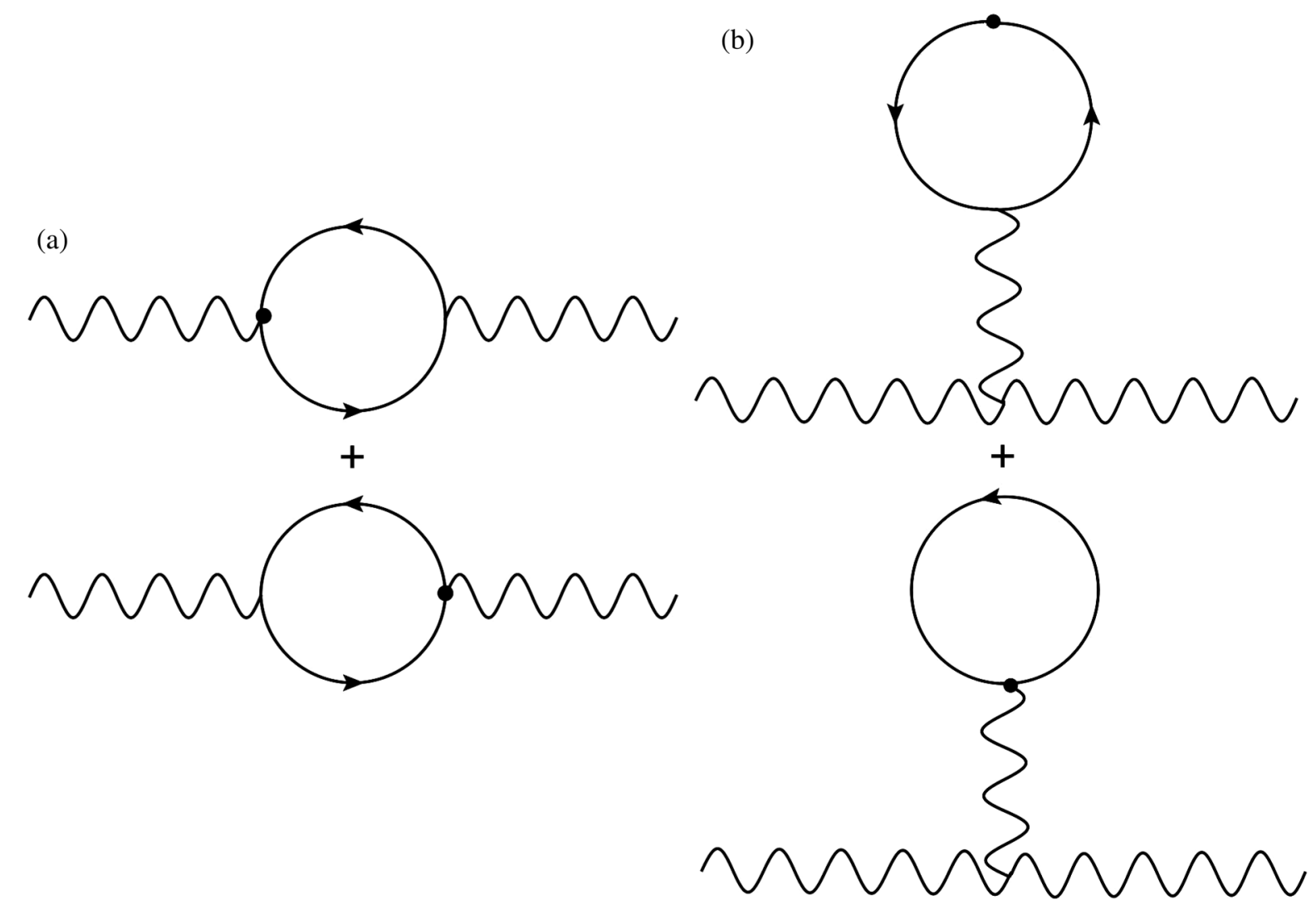

FIG. 3. Four diagrams that do not contribute to the radiatively induced gravitational Chern-Simons term. (a) Diagrams with a $b^{\mu}$-modified fermion-photon vertex, which leads to a term in which $b^{\mu}$ is contracted directly with one of the indices of the external graviton $h^{\mu \nu}$. (b) Pure tadpole diagrams.

invariance) because there are now three contributing diagrams - as opposed to the just two that were present in the Abelian gauge theory. Note that with a symmetric $k^{\prime \prime}$ integration, the diagram in Fig. 1(b) actually gives no net contribution to the Chern-Simons term; however, shifting the integration momentum by a multiple of $p_{1}$ does yield a surface term with a linear divergence. The calculation of the sum of the three diagrams proceeds along much the same lines as in the original papers on the gravitational contribution to the partially conserved axial current (PCAC) [38-40] (describing, for example, the potential rate of decay of a $\pi^{0}$ into two gravitons). The cubic divergences in the triangle diagrams automatically cancel, because they have opposite momentum routings and $\gamma_{5}$ is present. However, the cubic cancellation still leaves a residual linear divergence. In the Abelian case, the residual finite term in the sum of the two triangle diagrams was set by a $p_{1}$-dependent difference in the integration momenta $k$ and $k^{\prime}$; here in the gravitational calculation, the remaining linear divergence is set by the difference in $k$ and $k^{\prime}$. However, with a shift in the integration momentum $k^{\prime \prime}$ in the tadpole diagram, this linear divergence may also be canceled.

These results, for both kinds of gauge theories, can also be expressed in terms of the number of Ward identities that need to be satisfied. When the axial current vertex carries an external momentum (as it does in $\pi^{0}$ decay), there are two independent Ward identities, because there are two different external momenta to which the fermion loop matrix element must be transverse. Violations of gauge invariance must come from divergent loop integrals. Imposing the first Ward identity always forces the strongest naive divergence to vanish. However, there are still possible gauge symmetry violations coming from surface terms associated with the Feynman diagram divergences. In the electromagnetic case (with a naive linear divergence), there is one possible surface term, which may be adjusted to zero by choosing $k-k^{\prime}$. This enforces the second independent Ward identity. In the gravitational version, there are two possible surface terms (a linearly divergent one and a finite one), because the initial degree of divergence is cubic. Again however, by suitable choices of both $k-k^{\prime}$ and $k-k^{\prime \prime}$, both the surface terms may be eliminated, again making the matrix element transverse to both boson momenta.

The situation is somewhat different when the axial vector vertex originates from a constant Lorentz-violating background $b^{\mu}$. In that case, as we have noted, there is only one Ward identity to enforce, because the momenta $p_{1}$ and $p_{2}$ of the external gauge bosons are redundant. In the Abelian 
case, that means that $k-k^{\prime}$ may be chosen to be any multiple of $p_{1}$, yielding an undetermined Chern-Simons term. In the gravitational theory, there is still one nontrivial affine condition relating $k, k^{\prime}$, and $k^{\prime \prime}$ that is needed to ensure the linear divergence cancels. However, this means once again that there remains one undetermined parameter, and different choices of this parameter will produce different values for the induced $v^{\mu}$. Other regulators for the naively divergent diagrams introduce the ambiguity in other ways, just as in the Abelian theory. It may seem natural, therefore, to conclude that the coefficient of the induced gravitational Chern-Simons term should be entirely undetermined, just as in the Abelian case.

Before continuing, we shall pause briefly to point out why the only possible contributions to the induced Chern-Simons term actually come from the Feynman diagrams in Fig. 1, even though there are several other diagrams that can be constructed formally as contributions to the graviton twopoint function. The noncontributing diagrams are shown in Fig. 3. The two diagrams appearing in Fig. 3(a) have the Lorentz violation entering through the $b^{\mu}$-modified interaction vertex from Fig. 2. However, any diagram with a $b^{\mu}$ vertex could only give a contribution to the effective action with the Lorentz index of $b^{\mu}$ directly contracted with one of the external $h^{\mu \nu}$ metric modes - and such a term is not of the Chern-Simons form. Moreover, the other two diagrams in Fig. 3(b) are not one-particle irreducible. They have pure tadpole forms, with the intermediate graviton propagator necessarily carrying vanishing momentum, so no information about the momentum of the external gravitons can reach the divergent fermion loop. In any reasonable renormalization scheme, the sum of all the one-point graviton tadpole diagrams will be set to vanish (so that the nonfluctuating part of the metric $g^{\mu \nu}$ takes on its proper background value).

More details of the integrals, covering both the Abelian and QED cases, are given in the Appendix.

\section{RESOLVING THE PUZZLE}

The results of the previous section bring us back to the extremely puzzling point that while a $b^{\mu}$ term in the fermion sector is Lorentz violating, any proportional $v^{\mu}$ that is induced in the linearized gravity sector is not. It seems very counterintuitive that the quantum corrections at first order in $b^{\mu}$ should somehow "restore" the broken Lorentz symmetry, at least in the gravitational sector. There is no analogous symmetry restoration for the electromagnetic radiative corrections; the $k_{A F}^{\mu}$ violates the same spacetime symmetries as a $b^{\mu}$ term. Doubly puzzling is that while the gravitational Chern-Simons term avoids the Lorentz violation associated with $b^{\mu}$, it still has the same discrete symmetries (and hence the same $C P T$ violation) as the fermion sector term.

However, this paradoxical—or, at the least, extremely curious - behavior turns out to be an artifact of having used an oversimplified description of Lorentz violation in conjunction with gravity. In fact, the direct generation of a Lorentz-invariant radiative correction by a Lorentzviolating term in the fermion sector does not actually occur.

We have repeatedly noted that when the axial vector vertex insertion in the fermion propagator can carry a nonzero momentum, the number of constraints on a bosonic two-point function changes. If momentum can be exchanged with the axial vector background, then the momenta of the two attached bosons are not identical, and then there are two independent transverse Ward identities that must hold. Then the argument that the radiative corrections to the boson propagator must be at least $\mathcal{O}\left(p_{1}^{2}\right)$ is entirely correct, and a Chern-Simons term is excluded. Notice that insisting that the two separate Ward identities both hold when there is a net external momentum being inserted into the loop diagrams is equivalent to demanding that the Fourier transform of the effective Lagrange density be gauge invariant at the value of the external momentum in question. In fact, the only Fourier component of the (electromagnetic or gravitational) ChernSimons term that is invariant under gauge transformations is the zero-momentum component-which is just the integrated action. If a stronger form of gauge invarianceinvariance of the density $\mathcal{L}$, rather than just $S$-is required, then the coefficient $v^{\mu}$ in the gravitational sector must vanish.

It happens to be the case that Lorentz violation in a metric theory of gravity must arise spontaneously. Spontaneous Lorentz symmetry breaking is analogous to other, more familiar, types of spontaneous symmetry breaking. A bosonic field acquires a vacuum expectation value (vev), so that the vacuum state of the theory does not respect all the symmetries of the underlying Lagrangian. If the field with the vev has tensor indices, then the vev becomes a preferred tensor in the vacuum. Couplings of other fields to the symmetry-breaking field give rise to SME-type Lorentz-violating operators.

In a flat-space QFT like QED, Lorentz violation might arise spontaneously, or it might be explicit. In the latter case, the fundamental Lagrangian for the theory contains operators that violate Lorentz symmetry. Either possibility is internally consistent, although what we know about symmetry breaking in real physical systems may suggest that the spontaneous symmetry breaking might be more elegant. In a gravitational theory, however, matters are quite different. Only spontaneous symmetry breaking is possible; gravity theories with explicit Lorentz breaking turn out to be mathematically inconsistent. In a metric theory with explicit symmetry breaking, the Bianchi identities cannot be satisfied, and the theory fails [41].

The qualitative reason for the inconsistency is actually rather simple. The basic premise of a metric theory of gravitation is that test particles are moving along geodesics of a background spacetime configuration. Two particles with equal mass, beginning at the same point and moving with the same initial speeds, must follow exactly the same 
trajectory. There is no room for the dynamics to depend on anything else; there is no way to incorporate the spin and orientation dependences normally associated with the motion of different species of particles in a theory with explicitly broken rotation or boost symmetry. A preferred direction like $b^{\mu}$ cannot affect the motion of a fermion if the fermion's motion is entirely determined by the spacetime geometry that it is passing through. This argument holds even in a pure gravity theory, because gravity actually provides its own test quanta. Pure gravity theories have propagating gravitons, which in the linear theory are still effectively passing through a background geometry.

This geometric obstruction has spurred some interest in studying Lorentz violation in more general Finsler spacetimes. However, this work is still in its infancy; basic constructions, such as of a scalar field action or a spinor bundle have not yet been demonstrated. For the present purposes, we shall continue to suppose that gravitation is described by a metric theory like general relativity. The structure and consequences of a gravitational ChernSimons-like term in a Finsler geometry are matters far beyond the current state of understanding.

The fact that Lorentz violation in a metric theory of gravity must be spontaneous has definite phenomenalistic consequences. There must be additional fluctuating modes in the theory, which affect the physical observables in both the purely gravitational sector [42] as well as with mattergravity couplings [43]. The observation that the preferred $b^{\mu}$ is formed from the vacuum expectation value of vectorvalued fields on the spacetime manifold leads to a number of interesting conclusions and opens up new avenues of investigation. The vector field underlying $b^{\mu}$ may have a global structure related to the topology of the spacetime. Moreover, there will be additional quantized excitations which are coupled to the theory's fermions in the same way as $b^{\mu}$ itself.

However, what is important here is that the $b^{\mu}$ that appears in the Feynman rules in the gravitational theory cannot just be a fixed background vector. Instead, it is accompanied by additional fluctuating degrees of freedom. While the fluctuations themselves may be extremely small, the very fact that they must be possible changes the conceptual nature of the $b^{\mu}$ vertices. Because $b^{\mu}$ is the vev of a dynamical field, it has to be possible for there to be momentum exchange between the $b^{\mu}$ vertex and the gravitational field. When a nonzero momentum can enter the fermion loops at the $b^{\mu}$ insertion, there are going to be two nontrivial Ward identities, and the effective Lagrange density itself-not just the integrated effective actionmust be gauge invariant. This returns the theory to the original situation that was studied in the context of PCAC, in which the form of the radiative corrections is completely fixed. For the coefficient of the induced gravitational Chern-Simons term, the resulting unambiguous value is zero.

Thus the highly peculiar behavior of the radiative corrections - that there could be a Lorentz-invariant correction that is linear in the Lorentz-violating parameter $b^{\mu}$-has thus been avoided. The reason for this is that the arbitrariness of the Chern-Simons-type radiative corrections only exists when the Lorentz violation in the theory is explicit-which itself may be a rather unexpected result, although not a potentially paradoxical one. In fact, we may take the argument one step further and note that the when Lorentz-violating QED is studied in the (realistic) context of a background spacetime governed by general relativity, the $b^{\mu}$ in the fermion sector still has to be just one piece of a dynamical field. This means that the radiative-induced Chern-Simons term for the Abelian theory is also zero!

We have reached this level of understanding by drawing together earlier conclusions about the mathematical structures of different kinds of Lorentz-violating field theories. This further reinforces the observation that when basic symmetries such as Lorentz symmetry or $C P T$ are broken there may be some fairly subtle effects, qualitatively unlike those seen in more symmetric models-especially in regard to quantum corrections.

\section{APPENDIX: FERMION LOOP INTEGRALS}

To see how the cancellation between divergent terms works in the Lorentz-violating theories (both electromagnetic and gravitational) it is useful to inspect the loop integrals involved explicitly. For the QED case, the integration structure has been explored by a variety of methods in $[7,8,10]$, including the nonanalytic dependences on both $b^{\mu}$ and $p^{\mu}$. However, the key features for our analysis appear at leading order in both these quantities, so we shall limit ourselves to that order. At first order in $b^{\mu}$, the relevant terms in the photon self-energy are given by

$$
\begin{aligned}
\Pi^{\mu \nu}(p)= & -i q^{2} \int \frac{d^{4} k}{(2 \pi)^{4}} \operatorname{tr}\left\{\gamma^{\mu} \frac{i}{k-m} \gamma^{\nu} \frac{i}{k+\not p-m}\left(i \not \not \gamma_{5}\right) \frac{i}{k+\not p-m}\right. \\
& \left.+\gamma^{\nu} \frac{i}{k+\zeta \not p-m} \gamma^{\mu} \frac{i}{k+(\zeta+1) \not p-m}\left(i \not \not \gamma_{5}\right) \frac{i}{k+(\zeta+1) \not p-m}\right\} .
\end{aligned}
$$

The free parameter $\zeta$ describes the arbitrary momentum routing difference between the two contributing diagrams. 
The $p$-independent contribution to this self-energy is simply

$$
\Pi^{\mu \nu}(0)=-i q^{2} b^{\alpha} \int \frac{d^{4} k}{(2 \pi)^{4}} \operatorname{tr}\left\{\gamma^{\mu} \frac{k+m}{k^{2}-m^{2}} \gamma^{\nu} \frac{k+m}{k^{2}-m^{2}} \gamma_{\alpha} \gamma_{5} \frac{k+m}{k^{2}-m^{2}}+(\mu \leftrightarrow \nu)\right\} .
$$

This can be shown to vanish using explicit Dirac algebra calculations. However, following [7], it is simpler to notice that the contraction of the integral in (A2) with $b^{\alpha}$ is a twoindex object that is proportional to the Levi-Civita tensor $\epsilon^{\alpha \beta \gamma \delta} b_{\alpha}$, yet independent of $p$; no such object can be constructed, so the integral must vanish. Note that the involvement of the $\gamma_{5}$ (which is defined in terms of the Levi-Civita tensor) plays a key role in the cancellation; virtual fermions with opposite helicities contribute oppositely to the naively linearly divergent term.

As already noted, the term linear in the momentum vanishes for $\zeta=3+\xi=3$, which is the choice of momentum routing in the usual calculation of the QED chiral anomaly. The nonzero contribution then comes entirely from a surface term, which is given by the shifting the loop momentum in one diagram by $\xi p$,

$$
\begin{aligned}
\Pi^{\mu \nu}(p)= & q^{2} b^{\alpha} \int \frac{d^{4} k}{(2 \pi)^{4}} \operatorname{tr}\left\{\gamma^{\mu} \frac{1}{k-m} \gamma_{\alpha} \gamma_{5} \frac{1}{k-m} \gamma^{\nu} \frac{1}{k+\xi \not p-m}+\gamma^{\mu} \frac{1}{k+\xi \not p-m} \gamma_{\alpha} \gamma_{5} \frac{1}{k+\xi \not p-m} \gamma^{\nu} \frac{1}{k-m}\right\} \\
= & q^{2} b^{\alpha} \int \frac{d^{4} k}{(2 \pi)^{4}} \operatorname{tr}\left\{\gamma^{\mu} \frac{k+m}{k^{2}-m^{2}} \gamma_{\alpha} \gamma_{5} \frac{k+m}{k^{2}-m^{2}} \gamma^{\nu} \frac{k+m}{k^{2}-m^{2}} \xi \not p \frac{k+m}{k^{2}-m^{2}}\right. \\
& \left.+\gamma^{\mu} \frac{k+m}{k^{2}-m^{2}} \xi \not p \frac{k+m}{k^{2}-m^{2}} \gamma_{\alpha} \gamma_{5} \frac{k+m}{k^{2}-m^{2}} \xi \not p \frac{k+m}{k^{2}-m^{2}} \gamma^{\nu} \frac{k+m}{k^{2}-m^{2}}\right\} .
\end{aligned}
$$

The remaining divergence [coming from the terms in integrand that are proportional to $\left.k^{4} /\left(k^{2}+m^{2}\right)^{4}\right]$ in this surface term expression cancels [10], leaving a finite expression; the straightforward result is, at leading order in $p$,

$$
\Pi^{\mu \nu}(p)=-\frac{\xi q^{2}}{8 \pi^{2}} \epsilon^{\mu \nu \alpha \beta} b_{\alpha} p_{\beta}
$$

corresponding to the ambiguous $k_{A F}^{\mu}$.

The cancellations function similarly for the gravitational Chern-Simons term, but they are more elaborate, since the naive degree of divergences of the integrals involved are greater. However, it is not excessively difficult to get the potentially nonzero contribution to the radiatively generated $v^{\mu}$ in a form that closely mirror the results in the QED theory. The version of the calculation that arises in the PCAC context (with the axial vector insertion carrying nonzero momentum) is given in detail in [38], so we will concentrate on the case where the $b^{\mu}$ carries no momentum - the situation out of which a potentially ambiguous radiative correction could arise.

We will begin with the fermion tadpole diagram shown in Fig. 1(b), which does not contribute directly to the Chern-Simons term when the $k$-integration momentum is done symmetrically. If the external gravitons carry momentum $p$, and have polarization indices $(\mu, \nu)$ and $(\alpha, \beta)$, respectively, the Feynman rules give a self-energy tensor

$$
\Pi_{1 b}^{\mu \nu \alpha \beta}(p)=-\frac{i}{96} \int \frac{d^{4} k}{(2 \pi)^{4}} \operatorname{tr}\left\{\eta^{\mu \alpha} \gamma^{(\nu \beta \rho)}\left(2 p_{\rho}\right) \frac{i}{k}\left(i \not b \gamma_{5}\right) \frac{i}{k}\right\},
$$

recalling that we are using massless fermions for simplicity. With the symmetric integration, there is no contribution in (A6) to the gravitational Chern-Simons term, which is $\mathcal{O}\left(p^{3}\right)$. What is more, the massless integral, while superficially quadratically divergent, actually vanishes if the integration is done by dimensional regularization and evaluated at $d=4$. Note that the trace in (A6), combined with symmetric integration over $k$, gives an overall expression proportional to $\eta^{\mu \alpha} \epsilon^{\nu \beta \rho \delta} p_{\rho} b_{\delta}$, so this term, on its own, would (if it were not vanishing) satisfy the Ward identities for transversality in the indices $\nu$ and $\beta$.

The important part of the potentially ambiguous radiative correction in the gravitational theory really comes (as in the Abelian gauge theory) from the two triangle diagrams from Fig. 1(a). The sum of the diagrams looks similar to what appears in the QED version, but there is an additional momentum factor at each fermion-boson vertex. The sum of the two triangles, again with an undetermined difference $\zeta p$ between the integration momenta, is 


$$
\begin{aligned}
\Pi_{1 a}^{\mu \nu \alpha \beta}(p)= & \frac{i}{16} \int \frac{d^{4} k}{(2 \pi)^{4}} \operatorname{tr}\left\{\gamma^{\mu}(2 k+p)^{\nu} \frac{i}{k^{\gamma}} \gamma^{\alpha}(2 k+p)^{\beta} \frac{i}{k+\not p}\left(i \not \not \gamma \gamma_{5}\right) \frac{i}{k+\not \not}+\gamma^{\alpha}[2 k+(2 \zeta+1) p]^{\beta} \frac{i}{k+\zeta \not p} \gamma^{\mu}[2 k+(2 \zeta+1) p]^{\nu}\right. \\
& \left.\times \frac{i}{k+(\zeta+1) \not p}\left(i \not \not \gamma_{5}\right) \frac{i}{k+(\zeta+1) \not p}\right\} .
\end{aligned}
$$

As in (A2), the most divergent contribution $\Pi_{1 a}^{\mu \nu \alpha \beta}(0)$ (with a cubic divergence in this case) must vanish, since it has an impossible tensor structure-symmetric in $(\mu, \nu)$ and $(\alpha, \beta)$ and also proportional to the Levi-Civita tensor, but independent of $p$; this necessarily means $\Pi_{1 a}^{\mu \nu \alpha \beta}(0)=0$.

The quadratically divergent part of $\Pi_{1 a}^{\mu \nu \alpha \beta}(p)$ then automatically vanishes in the same way as the quadratic divergence in $\Pi_{1 b}^{\mu \nu \alpha \beta}(p)$, using Wick-rotated dimensional regularization at $d=4$.

What remains is a difference of two linearly divergent integrals, as in the QED case. Explicit calculation of the full integrals is extraordinarily tedious. However, it is possible to verify that $\zeta$ plays the same role in parametrizing the ambiguity in the gravitational theory as in the Abelian theory in a relatively straightforward way. This is done by differentiating $\Pi_{1 a}^{\mu \nu \alpha \beta}(p)$ with respect to $\zeta$, giving

$$
\begin{aligned}
& \left.\frac{d \Pi_{1 a}^{\mu \nu \alpha \beta}}{d \zeta}\right|_{\zeta=0}=\frac{i}{16} \int \frac{d^{4} k}{(2 \pi)^{4}} \operatorname{tr}\left\{\gamma^{\alpha}(2 p)^{\beta} \frac{1}{k^{\mu}} \gamma^{\mu}(2 k+p)^{\nu} \frac{1}{k+\not p} \not \gamma_{5} \frac{1}{k+\not p}+\gamma^{\alpha}(2 k+p)^{\beta} \frac{1}{\not k}(-\not \supset) \frac{1}{k} \gamma^{\mu}(2 k+p)^{\nu} \frac{1}{k+\not p} \not p \gamma_{5} \frac{1}{k+\not p}\right. \\
& +\gamma^{\alpha}(2 k+p)^{\beta} \frac{1}{k} \gamma^{\mu}(2 p)^{\nu} \frac{1}{k+\not p} \not \not \gamma_{5} \frac{1}{k+\not p}+\gamma^{\alpha}(2 k+p)^{\beta} \frac{1}{k^{\prime}} \gamma^{\mu}(2 k+p)^{\nu} \frac{1}{k+\not p}\left[(-\not p) \frac{1}{k+\not p} \not b \gamma_{5}\right. \\
& \left.\left.+\not \gamma_{5} \frac{1}{k+\not p}(-\not p)\right] \frac{1}{k+\not p}\right\} \text {. }
\end{aligned}
$$

Taking into account the fact that the parts of this expression which come from terms with quadratic and higher divergences must vanish, (A8) simplifies to

$$
\begin{aligned}
\left.\frac{d \Pi_{1 a}^{\mu \nu \alpha \beta}}{d \zeta}\right|_{\zeta=0}= & -\frac{i}{16} \int \frac{d^{4} k}{(2 \pi)^{4}} \operatorname{tr}\left\{\gamma^{\alpha} p^{\beta} \frac{1}{k^{\prime}} \not \frac{1}{k^{\mu}} \gamma^{\mu} p^{\nu} \frac{1}{k+\not p} \not \phi \gamma_{5} \frac{1}{k+\not p}\right. \\
& \left.+\gamma^{\alpha} p^{\beta} \frac{1}{k^{\prime}} \gamma^{\mu} p^{\nu} \frac{1}{k+\not p}\left[\not p \frac{1}{k+\not p} \not p \gamma_{5}+\not b \gamma_{5} \frac{1}{k+\not p} \not p\right] \frac{1}{k+\not p}\right\} .
\end{aligned}
$$

This has the same functional form as the equivalent derivative of (A1), in the massless limit, $d \Pi^{\mu \nu} /\left.d \zeta\right|_{\zeta=0}$. There are two extra powers of the momentum in (A9), which just correspond to the additional derivatives in the gravitational ChernSimons term. Since a linear shift in $\zeta$ in the Abelian theory creates a proportional shift in the induced $k_{A F}^{\mu}$, a similar change in the momentum routing in the gravitational theory would produce an equivalent linear shift in $v^{\mu}$.

[1] O. W. Greenberg, Phys. Rev. Lett. 89, 231602 (2002).

[2] D. Colladay and V. A. Kostelecký, Phys. Rev. D 55, 6760 (1997).

[3] D. Colladay and V. A. Kostelecký, Phys. Rev. D 58, 116002 (1998).

[4] V. A. Kostelecký and N. Russell, Rev. Mod. Phys. 83, 11 (2011); updated as arXiv:0801.0287v12.

[5] S. M. Carroll, G. B. Field, and R. Jackiw, Phys. Rev. D 41, 1231 (1990).

[6] S. Coleman and S. L. Glashow, Phys. Rev. D 59, 116008 (1999).
[7] R. Jackiw and V. A. Kostelecký, Phys. Rev. Lett. 82, 3572 (1999).

[8] M. Pérez-Victoria, Phys. Rev. Lett. 83, 2518 (1999).

[9] J. M. Chung, Phys. Lett. B 461, 138 (1999).

[10] J. M. Chung and P. Oh, Phys. Rev. D 60, 067702 (1999).

[11] W. F. Chen, Phys. Rev. D 60, 085007 (1999).

[12] J. M. Chung, Phys. Rev. D 60, 127901 (1999).

[13] G. E. Volovik, J. Exp. Theor. Phys. Lett. 70, 1 (1999).

[14] L.-H. Chan, arXiv:hep-ph/9907349.

[15] G. Bonneau, Nucl. Phys. B593, 398 (2001). 
[16] M. Chaichian, W. F. Chen, and R. G. Felipe, Phys. Lett. B 503, 215 (2001).

[17] M. Pérez-Victoria, J. High Energy Phys. 04 (2001) 032.

[18] O. A. Battistel and G. Dallabona, J. Phys. G 27, L53 (2001).

[19] A. A. Andrianov, P. Giacconi, and R. Soldati, J. High Energy Phys. 02 (2002) 030.

[20] B. Altschul, Phys. Rev. D 69, 125009 (2004).

[21] B. Altschul, Phys. Rev. D 70, 101701(R) (2004).

[22] T. Mariz, J. R. Nascimento, E. Passos, and R. F. Ribeiro, Phys. Rev. D 70, 024014 (2004).

[23] T. Mariz, J. R. Nascimento, A. Y. Petrov, L. Y. Santos, and A. J. da Silva, Phys. Lett. B 661, 312 (2008).

[24] M. Gomes, T. Mariz, J. R. Nascimento, E. Passos, A. Y. Petrov, and A. J. da Silva, Phys. Rev. D 78, 025029 (2008).

[25] J. C. C. Felipe, A. R. Vieira, A. L. Cherchiglia, A. P. B. Scarpelli, and M. Sampaio, Phys. Rev. D 89, 105034 (2014).

[26] J. F. Assunção, T. Mariz, J. R. Nascimento, and A. Y. Petrov, J. High Energy Phys. 08 (2018) 072.

[27] S. Alexander and N. Yunes, Phys. Rev. Lett. 99, 241101 (2007).

[28] T. L. Smith, A. L. Erickcek, R. R. Caldwell, and M. Kamionkowski, Phys. Rev. D 77, 024015 (2008).
[29] S. Alexander, L. S. Finn, and N. Yunes, Phys. Rev. D 78, 066005 (2008).

[30] R. Jackiw and S.-Y. Pi, Phys. Rev. D 68, 104012 (2003).

[31] D. Guarrera and A. J. Hariton, Phys. Rev. D 76, 044011 (2007).

[32] V. A. Kostelecký and R. Lehnert, Phys. Rev. D 63, 065008 (2001).

[33] D. Colladay and V. A. Kostelecký, Phys. Lett. B 511, 209 (2001).

[34] J. S. Bell and R. Jackiw, Nuovo Cimento A 60, 47 (1969).

[35] R. Jackiw, Int. J. Mod. Phys. B 14, 2011 (2000).

[36] B. Altschul, Phys. Rev. D 73, 036005 (2006).

[37] L. Alvarez-Gaumé and E. Witten, Nucl. Phys. B234, 269 (1984).

[38] T. Kimura, Prog. Theor. Phys. 42, 1191 (1969).

[39] R. Delbourgo and A. Salam, Phys. Lett. 40B, 381 (1972).

[40] R. Delbourgo, J. Phys. A 10, L237 (1977).

[41] V. A. Kostelecký, Phys. Rev. D 69, 105009 (2004).

[42] Q. G. Bailey and V. A. Kostelecký, Phys. Rev. D 74, 045001 (2006).

[43] V. A. Kostelecký and J. D. Tasson, Phys. Rev. D 83, 016013 (2011). 